\title{
Crystallization behavior and higher-order structure in miscible crystalline/crystalline polymer blends
}

\author{
Fuminori Arai, Kazuya Shinohara, Noriaki Nagasawa, Hiroki Takeshita, Katsuhiko Takenaka, Masamitsu Miya \\ and Tomoo Shiomi \\ The crystallization behavior and higher-order structures of crystalline/crystalline miscible polymer blends composed of \\ poly(vinylidene fluoride) (PVDF) and isotactic-poly(methyl methacrylate) (i-PMMA) were investigated using small-angle X-ray \\ scattering (SAXS) and wide-angle X-ray diffraction techniques, as well as differential scanning calorimetry. When only the PVDF \\ component was crystallized, the overall crystallization rate decreased with increasing $i$-PMMA composition and exhibited a \\ bell-shaped temperature dependence. The long period increased with increasing $i$-PMMA content up to $0.5 \mathrm{wt}$. fr. and then \\ decreased at $0.4 \mathrm{wt}$. fr. In the subsequent crystallization of $i$-PMMA, the overall crystallization rate was enhanced compared \\ with that of the neat $i$-PMMA; this enhancement was attributed to the role of PVDF as a nucleating agent because the \\ nucleation induction period was shortened. From the SAXS profiles, it was concluded that $i$-PMMA crystallized between the \\ PVDF crystalline lamellae and that the long period between the PVDF crystalline lamellae was consequently expanded. For the \\ crystallization of $i$-PMMA in the blend that contained $0.7 \mathrm{wt}$. fr. of PVDF, both the Avrami index and crystallinity were \\ depressed, which suggested that the crystallization of $i$-PMMA was confined by the PVDF crystalline lamellae. \\ Polymer Journal (2013) 45, 921-928; doi:10.1038/pj.2013.5; published online 13 February 2013
}

Keywords: crystallization; crystallization rate; lamellar structure; polymer blend; SAXS

\section{INTRODUCTION}

Over the past few decades, the crystallization behavior in miscible crystalline/amorphous polymer blends has been extensively investigated $^{1}$. During crystallization in such blend systems, the amorphous component behaves as a diluent such that it diffuses away from the crystal growth front. However, the diffusion length may not be as long in typical polymer blends because the molecular mobility is quite low. Consequently, the amorphous component may be incorporated within the interlamellar region. This interlamellar inclusion has been observed in blends such as poly(ethylene oxide)/atactic-poly(methyl methacrylate) (a-PMMA $)^{2}$, poly $(\varepsilon$-caprolactone $) /$ poly(vinyl chloride $)^{3}$, polycarbonate (PC)/poly $(\varepsilon \text {-caprolactone })^{4}$, poly(vinylidene fluoride) (PVDF)/poly(3-hydroxybutyrate $)^{5}$ and $\operatorname{PVDF} / \operatorname{poly}(1,4-$ butylene adipate $)^{6,7}$.

According to the Keith-Padden theory, the inclusion or exclusion of the amorphous component is dominated by the relationship between the crystallization rate and the molecular diffusion rate . $^{8}$ When the crystallization rate is sufficiently low and the mobility is sufficiently high, the amorphous component can be excluded from the interlamellar region, even in miscible blends. Saito et al. have experimentally confirmed the exclusion effect using PVDF/a-PMMA blends $s^{9,10}$. These authors reported that the spherulite of PVDF adopts a coarse morphology when the crystallization rate is relatively low, whereas it becomes more compact during rapid crystallization because the amorphous a-PMMA component diffuses from the interlamellar region to larger spaces, such as interfibrillar and/or inter-spherulite regions.

The amorphous component in miscible polymer blends usually decreases crystallization rates through the effect of the composition dependence of the glass transition temperature and through diluent effects $^{11}$. Furthermore, it has also been reported that nucleation is enhanced by the spinodal decomposition during crystallization that simultaneously occurs with liquid-liquid phase separation ${ }^{12-14}$.

When both components are crystallizable, two crystallization mechanisms are possible: simultaneous crystallization and subsequent crystallization. Some unique morphologies, such as concentrically growing or interpenetrating spherulites, have recently been reported to occur via simultaneous crystallization ${ }^{15-25}$. In the subsequent crystallization, the effect of the pre-existing crystal structure on the crystallization kinetics and the resulting lamellar structure has not yet been sufficiently discussed ${ }^{4-7}$.

In this study, the crystallization behavior and the higher-order structure were investigated using miscible crystalline/crystalline polymer blends of PVDF/isotactic-PMMA (i-PMMA). In this blend system, two-step crystallization can be performed because of the considerably slower crystallization rate of $i$-PMMA, which depends on 
its tacticity ${ }^{26-28}$, than that of PVDF. The $i$-PMMA used in this study requires at least several weeks to complete its crystallization, whereas PVDF requires only a few hours at most. Therefore, during the crystallization of PVDF, the crystallization of $i$-PMMA can be almost completely neglected, and $i$-PMMA behaves similar to an amorphous component at any crystallization temperature. The subsequent crystallization of $i$-PMMA can be performed under the pre-existing crystalline lamellae structure after the complete crystallization of PVDF.

In this paper, the lamellar structure containing amorphous $i$-PMMA formed by the crystallization of PVDF in the blends will be presented first, along with its crystallization and melting behaviors. Then, the crystallization kinetics and the final higher-order structure of the crystallization of the $i$-PMMA under the pre-existing PVDF crystals will be described.

\section{MATERIALS AND METHODS}

PVDF $\left(M_{\mathrm{w}}=60000\right)$ and $i$-PMMA $\left(M_{\mathrm{w}}=25000\right)$ were purchased from Polyscienses Inc. (Warrington, PA, USA) and Polymer Source Inc. (Dorval, Quebec, Canada), respectively. According to the information provided by the manufacturers, the glass transition temperatures of PVDF and $i$-PMMA are approximately $-40^{\circ} \mathrm{C}$ and $50^{\circ} \mathrm{C}$, respectively, and the tacticity of $i$-PMMA is $>98 \%$. The PVDF/i-PMMA blend samples with various blend compositions were prepared by solution casting from $\mathrm{N}, \mathrm{N}$-dimethylformamide solutions (approximately $5 \mathrm{wt} \%$ ) at $30^{\circ} \mathrm{C}$ for $24 \mathrm{~h}$ under vacuum.

Here, the miscibility of the PVDF/i-PMMA blends is noted. Roerdink and Challa observed a single glass transition and a negative interaction parameter for both blends of PVDF with $i$-PMMA and syndiotactic-PMMA (s-PMMA ${ }^{27}$. On the other hand, Sasaki et al. observed lower critical solution temperature (LCST)-type phase diagrams for blends of both PVDF/i-PMMA and PVDF/s$\mathrm{PMMA}^{28}$. The authors suggested that PVDF was phase separated below the melting temperature, $T_{\mathrm{m}}$ (approximately $450 \mathrm{~K}$ ), of PVDF in the $i$-PMMA composition range of $0.3-0.7$; this conclusion was based on their experimental results in which the cloud point line was overlapped with the $T_{\mathrm{m}}$ line, and the crystallization rate was almost independent of the blend composition in the range of $0-0.3$ of $i$-PMMA. The miscibility of PVDF/i-PMMA should depend on both the molecular weight of the components and the tacticity of $i$-PMMA. The molecular weights of the samples used by the authors were $M_{\mathrm{W}}=26.0$ $\times 10^{5}$ and $1.6 \times 10^{5}$ for PVDF and $i$-PMMA, respectively, and the isotactic content of $i$-PMMA was 0.93 . The molecular weights of the PVDF and $i$ PMMA used in this study are one-order of magnitude less than those used by Sasaki et al., and the isotactic contents are not considerably different from each other. In addition, as described in the Results and Discussion section, our blends show clear dependencies of both the crystallization rate and the melting point depression on the composition of the blends. Therefore, the blends used in this study are melt-miscible, at least in the investigated temperature and composition ranges.

The crystallization and the melting behavior of PVDF without $i$-PMMA crystallization were first observed by differential scanning calorimetry using a Perkin-Elmer Pyris 1 (Perkin-Elmer, Waltham, MA, USA) with an external water cooler maintained at $8^{\circ} \mathrm{C}$. Approximately $10 \mathrm{mg}$ of the blend sample in an aluminum pan was initially melted at $200^{\circ} \mathrm{C}$ (which is substantially higher than the melting point of both PVDF, $T_{\mathrm{m} \_\mathrm{PVDF}}=170^{\circ} \mathrm{C}$, and $i$-PMMA, $T_{\mathrm{m} \_} i$ PMMA $=144^{\circ} \mathrm{C}$ ) for $10 \mathrm{~min}$. The melted samples were quenched at the desired crystallization temperatures for PVDF, $T_{\mathrm{C}_{-} \mathrm{PVDF}}$, at a rate of $40^{\circ} \mathrm{C} \mathrm{min}^{-1}$. After completing the PVDF crystallization (for $30 \mathrm{~min}$ to $24 \mathrm{~h}$ ), its melting behavior was investigated by heating it from $T_{\mathrm{C}_{-} \mathrm{PVDF}}$ to $200^{\circ} \mathrm{C}$ at a rate of $10^{\circ} \mathrm{C} \mathrm{min}{ }^{-1}$.

To investigate the crystallization of $i$-PMMA under the pre-existing PVDF crystalline morphology, the following two-step crystallizations were performed: the samples that were annealed at $200{ }^{\circ} \mathrm{C}$ for $10 \mathrm{~min}$ were first quenched to several $T_{\mathrm{C}_{\perp} \mathrm{PVDF}} \mathrm{S}$ for the isothermal crystallization of PVDF (the first crystallization step), and then, these samples were further quenched to a fixed crystallization temperature of $100^{\circ} \mathrm{C}$ for the crystallization of $i$-PMMA (the second crystallization step).
As there are some operational difficulties in evaluating the crystallization behavior of $i$-PMMA using thermal analysis because of its extremely slow crystallization rate, wide-angle X-ray diffraction (WAXD) analyses were performed to estimate the crystallinity of $i$-PMMA as a function of time. These measurements were performed using a Rigaku NanoViewer (Rigaku, Tokyo, Japan) operating with $\mathrm{Cu} \mathrm{K} \alpha$ radiation $(\lambda=0.154 \mathrm{~nm})$ under an applied voltage of $45 \mathrm{kV}$ and a current of $60 \mathrm{~mA}$. The diffraction patterns were collected with an imaging plate and sector averaged to obtain one-dimensional WAXD profiles. As the obtained WAXD profiles contain the profiles of both PVDF and $i$-PMMA, the contribution of each species was extracted by curvefitting based on the peak positions of the respective neat polymers. The crystallinities at each crystallization time were calculated from the ratio of the crystalline peak areas to the amorphous halo.

To evaluate the higher-order structure of the crystal lamellae, small-angle $\mathrm{X}$-ray scattering (SAXS) measurements were performed using a synchrotron radiation source at beamline BL-6A of the Photon Factory at the High Energy Accelerator Research Organization (KEK), Tsukuba, Japan ${ }^{29}$. The scattered X-rays were detected using a CCD camera system (C7300: Hamamatsu Photonics KK, Hamamatsu, Japan) with an image intensifier. The scattering vector, which is defined as $q=(4 \pi / \lambda) \sin (\theta / 2)$, where $\theta$ and $\lambda$ are the scattering angle and the wavelength, respectively, was calibrated based on the diffraction pattern of silver behenate $(d=5.85 \mathrm{~nm})$.

\section{RESULTS AND DISCUSSION}

\section{Crystallization of PVDF during the first crystallization step}

During the crystallization of PVDF in the blends with $i$-PMMA, $i$-PMMA behaves as an amorphous component because of its extremely slow crystallization rate. Therefore, it is expected that the crystallization behavior of PVDF should be the same as that of a typical crystalline/amorphous blend. The melting behavior of PVDF was investigated first. As many researchers have reported, PVDF has several types of crystalline forms depending on the crystallization condition. During the melt crystallization process, the $\alpha$-phase crystalline structure of PVDF is known to be the most stable structure $^{30}$. As described later (Figure 6), the WAXD experiments confirmed that the $\alpha$-phase of the PVDF crystal is formed in this study. Figure 1a presents Hoffman-Weeks plots of the samples crystallized during the first crystallization step at several $T_{\mathrm{C}_{-}}$PVDFS The equilibrium melting temperature, $T_{\mathrm{m}}^{\circ}$, of PVDF in the blends can be estimated by extrapolating the plots of $T_{\mathrm{m}} \mathrm{vs} T_{\mathrm{c}}$ to the $T_{\mathrm{m}}=T_{\mathrm{c}}$ line. Melting point depression in a general crystalline/amorphous blend has been described by Nishi and Wang as follows:

$$
\frac{1}{v_{1}}\left(\frac{1}{T_{\mathrm{m}}^{\circ}}-\frac{1}{T_{\mathrm{m} 0}^{\circ}}\right)=-\left(\frac{B V_{2 \mathrm{u}}}{\Delta H_{2 \mathrm{u}}}\right)\left(\frac{v_{1}}{T_{\mathrm{m}}^{\circ}}\right),
$$

where $v_{1}$ is the volume fraction of the amorphous component, $T_{\mathrm{m} 0}{ }^{\circ}$ is the equilibrium melting temperature of the neat crystalline polymer, $V_{2 u}$ is the molar volume of the crystalline component, and $\Delta H_{2 u}$ is the heat of fusion per mole of the repeating unit of the crystalline component. $B$ is the so-called interaction energy density and is expressed by $B=\chi_{12}(R T) / V_{1 \mathrm{u}}$, where $\chi_{12}$ is the Flory-Huggins interaction parameter, $R$ is the gas constant and $V_{1 \mathrm{u}}$ is the molar volume of the amorphous component. The plots in Figure $1 \mathrm{~b}$ indicate a linear relationship between $T_{\mathrm{m} 0}^{\circ}-T_{\mathrm{m}}^{\circ}$ and $v_{1}^{2}$, as expected in typical miscible crystalline/amorphous blends.

The crystallization behavior of PVDF in the blends was estimated using differential scanning calorimetry thermal analysis. The overall crystallization rates, $G_{\mathrm{PVDF}}$, which are defined as the reciprocal of the half-time $t_{1 / 2}$ (time to achieve $50 \%$ of the final crystallinity), are plotted against $T_{\mathrm{c} \_ \text {PVDF }}$ in Figure 2. In general, the crystallization rate of miscible polymer blends consists of a $T_{\mathrm{g}}$-dependent molecular transport term and a nucleation term with a composition 

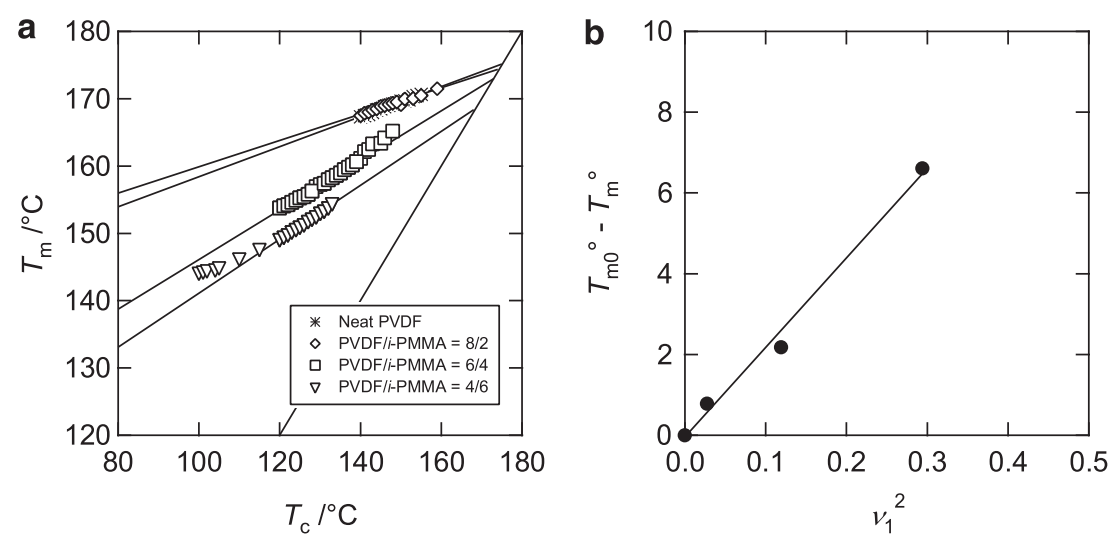

Figure 1 Hoffman-Weeks plots for the neat and blended poly(vinylidene fluoride) (PVDF) in the first crystallization step (a) and melting point depression for PVDF in blends with amorphous isotactic-poly(methyl methacrylate) ( $i$-PMMA) (b).

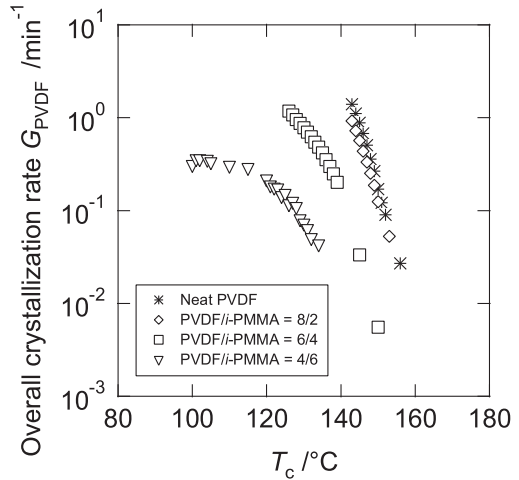

Figure 2 Crystallization temperature dependence of the overall crystallization rates for the neat and blended poly(vinylidene fluoride) (PVDF) during the first crystallization step.

contribution, and it can be expressed as

$$
G=v_{2} G_{0} \exp \left(-\frac{u^{*}}{R\left(T_{\mathrm{c}}-T_{\infty}\right)}\right) \exp \left(-\frac{K_{\mathrm{g}}}{T_{\mathrm{c}} \Delta T_{\mathrm{c}} f}+\frac{2 \sigma T_{\mathrm{m}}^{\circ} \ln v_{2}}{b \Delta H \Delta T_{\mathrm{c}}}\right),
$$

where $v_{2}$ is the volume fraction of the crystalline polymer in the blend, $G_{0}$ is a pre-exponential factor, $u^{*}$ is the activation energy for reputation in the melt, $T_{\infty}$ is the temperature of cessation of molecular motion (often taken at $T_{\mathrm{g}}-30{ }^{\circ} \mathrm{C}$ ), $K_{\mathrm{g}}$ is the nucleation constant, $\Delta \mathrm{T}_{\mathrm{c}}=T_{\mathrm{m}}^{\circ}-T_{\mathrm{c}}$ is the degree of supercooling, $f$ is a dimensionless correction factor given by $2 T_{\mathrm{c}} /\left(T_{\mathrm{m}}^{\circ}+T_{\mathrm{c}}\right), \sigma$ is the lateral surface-free energy of the crystalline lamella, $b$ is the thickness of a crystal lattice cell in the growth direction and $\Delta \mathrm{H}$ is the heat of fusion per unit volume for a perfect crystal.

As the contributions of the transport and nucleation terms to the crystallization rate are inversely related to $T_{\mathcal{C}}$, the crystal growth rate of polymers exhibits a bell-shaped temperature dependence between the melting point and the glass transition temperature. Uemoto and Okui suggested that the $T_{\mathrm{cmax}} / T_{\mathrm{m}}^{\circ}$ is approximately $5 / 6$ in general polymers, where $T_{\mathrm{cmax}}$ is the temperature that yields the maximum growth rate ${ }^{31}$. Although the crystallization rate shown in Figure 2 is not the growth rate but is instead the overall rate, $T_{\text {cmax }}$ in the blend having 0.4 wt. fr. of PVDF is approximately $100^{\circ} \mathrm{C}$, which is close to $95^{\circ} \mathrm{C}$, which was predicted using the $T_{\mathrm{m}}^{\circ}$ in Figure $1 \mathrm{a}$.
The crystallization rate in miscible blends at a given $T_{\mathrm{c}}$ is governed by the dependencies of both the dilution and glass transition effects on the composition, as shown in equation (2). Although it is difficult to compare the $G_{\mathrm{PVDF}}$ at exactly the same $T_{\mathrm{c} \_ \text {PVDF }}$ among all the blends because the experimental temperature range varies with the blend composition, as shown in Figure 2, G $G_{\mathrm{PVDF}}$ decreases as the amorphous $i$-PMMA content increases at a constant $T_{\mathrm{c} \text { PVDF }}$ The glass transition temperatures for the neat $i$-PMMA and PVDF are approximately $50{ }^{\circ} \mathrm{C}$ and $-40^{\circ} \mathrm{C}$, respectively, and the blend that contains 0.4 wt. fr. of PVDF exhibits its maximum crystallization rate at approximately $100{ }^{\circ} \mathrm{C}$. Therefore, it is suggested that the glass transition effect is not substantial at the $T_{\mathrm{c}}=128-160^{\circ} \mathrm{C}$ investigated in this study and that the dilution effect is a primary factor for decreasing the crystallization rate with the composition.

The isothermal crystallization kinetics of PVDF in the blends were analyzed using the well-known Avrami equation. The relative degree of crystallinity, $X_{\mathrm{t}}$, at the crystallization time $t$ is expressed as

$$
X_{\mathrm{t}}=1-\exp \left(-\mathrm{Kt}^{n}\right),
$$

where the exponent $n$ is an Avrami index that depends on the nucleation and growth dimension and the parameter $K$ is an overall crystallization rate constant. equation (3) gives

$$
\frac{d\left(1-X_{\mathrm{t}}\right)}{d(\ln t)}=n\left(1-X_{\mathrm{t}}\right) \ln \left(1-X_{\mathrm{t}}\right) \text {. }
$$

Equation (4) reveals that the curve of $1-X_{\mathrm{t}}$ vs $\ln t$ should fall on a master curve by some shift along the $\ln t$ axis when $K$ is independent of $t$ and $n$ is constant. Figure 3 a presents the plots of $1-X_{\mathrm{t}}$ vs $\ln t$. The shape of the curves is almost the same for all the samples, including the neat PVDF, and the optimal values of $n$ for these curves is approximately 3 , as determined by comparing the curves calculated using $n=1,2$ and 3 in Figure $3 c$. This result reveals three-dimensional crystal growth in this system because heterogeneous nucleation is believed to occur without any special treatment.

SAXS experiments were used to determine whether the amorphous component $i$-PMMA is included in the interlamellar region or excluded from this region during the crystallization of PVDF. The Lorentz-corrected SAXS profiles at each $T_{\mathrm{C} \_ \text {PVDF }}$ and the corresponding one-dimensional correlation function $K(r)$ obtained by the inverse Fourier transform of the SAXS profiles are shown in Figure 4. The peak position $q_{\max }$ in the SAXS profile yields an interlamellar spacing (distance between crystal lamellae) or the long period $d$ by $d=2 \pi / q_{\max }$. The correlation distance, $r$, at the first maximum of $K(r)$ 

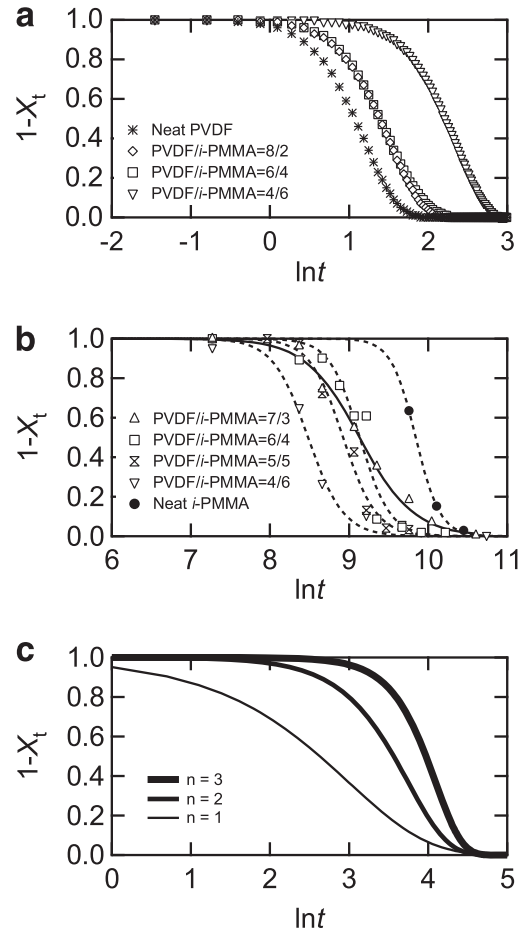

Figure 3 Avrami analyses for poly(vinylidene fluoride) (PVDF) crystallized during the first crystallization step (a) and for isotactic-poly(methyl methacrylate) ( $i$-PMMA) during the second crystallization step (b). The curves in (c) were calculated using equation 3 with $n=1,2$ and 3 .

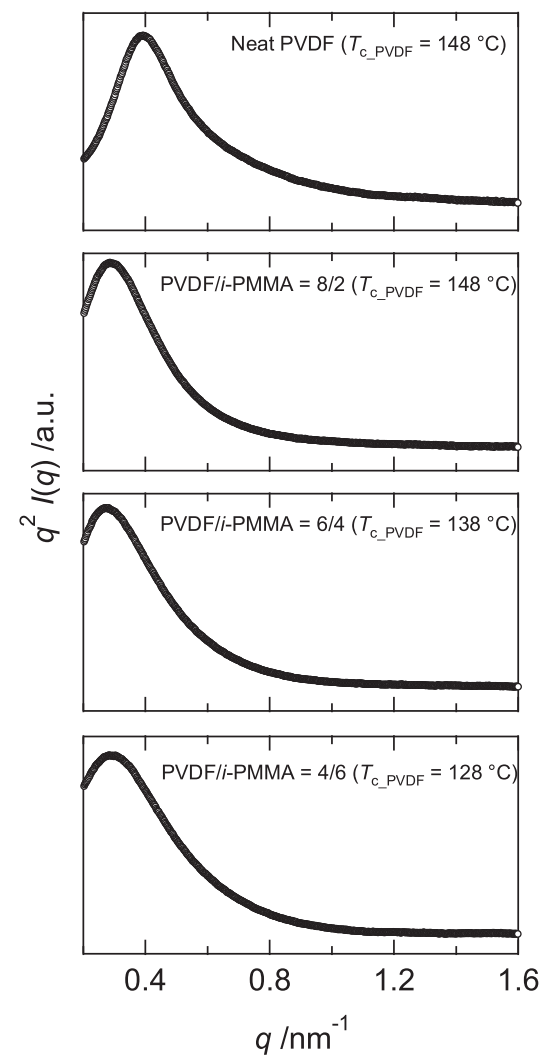

also represents the long period. The position of the first maximum of $K(r)$ cannot be clearly detected in the blend that contains $0.4 \mathrm{wt}$. fr. of PVDF. Therefore, the values of $d$ were estimated from $q_{\max }$ because the peak position in the SAXS profiles yields almost the same long period as that obtained from the correlation functions for the neat PVDF and the blends containing 0.8 and 0.6 wt. fr. of PVDF. The values of $d$ are plotted against the blend composition in Figure 5. The darker symbols represent the data at higher $T_{\text {c PVDF }}$ In the correlation function shown in Figure 4, the intersection between the straight line extrapolated from the so-called self-correlation triangle and the horizontal base line represents the thinner layer thickness, $L 1^{32}$. The obtained value of $L 1$ is assigned to the crystalline PVDF layer because the crystallinity of PVDF is estimated to be $<50 \%$ from the WAXD experiments, which are described later. In addition, the thickness of the amorphous layer, $L 2$, can be estimated as $L 2=d-L 1$. The obtained $L 1$ and $L 2$ are also plotted in Figure 5. $L 1$ is almost independent of the blend composition, whereas $d$ and $L 2$ increase with increasing $i$-PMMA content in the PVDF-rich blends. This result suggests that the amorphous $i$-PMMA component was entrapped between the PVDF lamellae. However, in the blends containing a PVDF content $<0.5$, the amorphous layer thickness, L2, slightly decreases as the $i$-PMMA content increases. In a miscible blend containing two crystalline polymers, PVDF and poly(1,4-butylene adipate), Liu et al. observed that the long period of PVDF increased with the poly(1,4-butylene adipate) content, leveled off at $30 \mathrm{wt} \%$ of poly (1,4-butylene adipate) and then decreased in the $60 \mathrm{wt} \%$ blend at the temperature where only PVDF crystallized ${ }^{5,6}$. The authors concluded that the interlamellar inclusion and exclusion behavior of the amorphous component was governed by the crystal growth rate
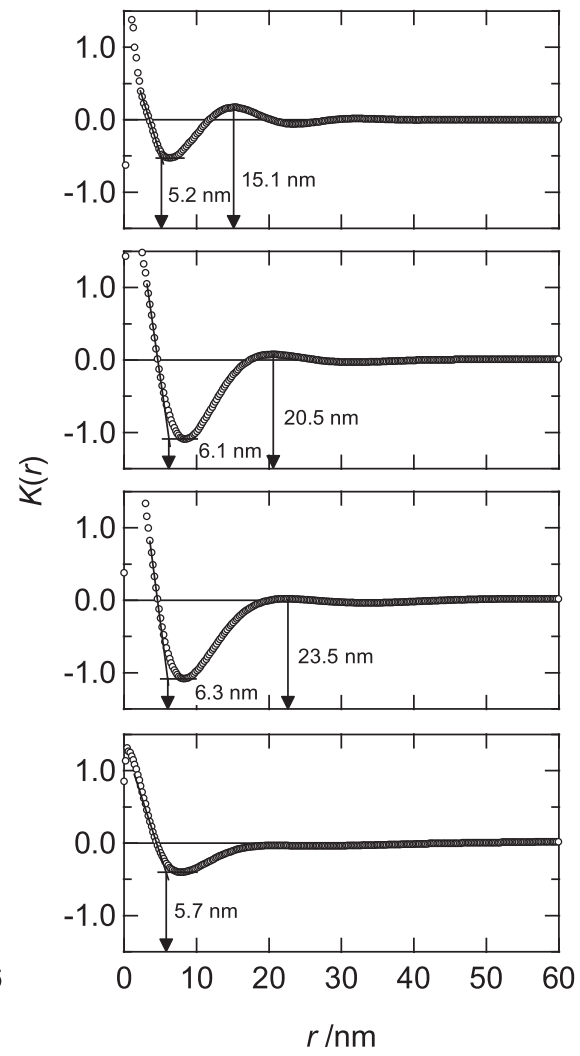

Figure 4 Lorentz-corrected small-angle X-ray scattering (SAXS) profiles (left) and the corresponding one-dimensional correlation functions (right) for the neat and blended poly(vinylidene fluoride) (PVDF) during the first crystallization step. 
relative to the diffusive mobility of the amorphous component. In the blends used in this study, as shown in Figure 2, the crystallization rate decreases with increasing $i$-PMMA content. In addition, as described above, the glass transition effect is not substantial, at least at temperatures considerably $>100{ }^{\circ} \mathrm{C}$. Therefore, there is sufficient time for the exclusion of $i$-PMMA from the interlamellar region in the blends containing a PVDF content $<0.5$.

For the blends crystallized at a representative $T_{\mathrm{c} \text { PVDF }}$ for each blend, WAXD experiments were performed to confirm the crystalline form and the crystallinity, $X_{\mathrm{c}_{-} \text {PVDF }}$ of PVDF during the first crystallization step. Figures $6 \mathrm{a}-\mathrm{c}$ present examples of the obtained WAXD profiles. The peak positions of these profiles were consistently attributed to the $\alpha$-phase for all the samples. The $X_{\mathrm{c} \_ \text {PVDF }}$ normalized

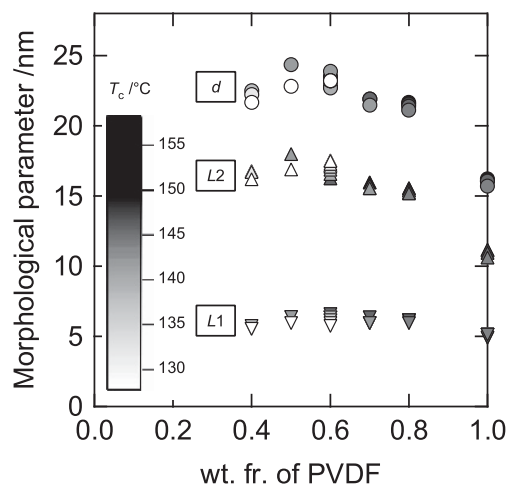

Figure 5 Dependence of the morphological parameters on the composition estimated from the small-angle X-ray scattering (SAXS) experiments for the samples crystallized during the first crystallization step. The circles and uppointing and down-pointing triangles indicate $d, L 2$ and $L 1$, respectively. The darker symbols indicate higher crystallization temperatures. to PVDF content was calculated from the areas of the crystal peaks and the amorphous halos of PVDF; these values are summarized in Table 1.

\section{Crystallization of $i$-PMMA during the second crystallization step} under pre-existing PVDF lamellae

The samples listed in Table 1 were further quenched from the respective $T_{\mathrm{C} \text { PVDF }}$ to $100{ }^{\circ} \mathrm{C}$ for the second crystallization step. The development of the $i$-PMMA crystallinity under the pre-existing PVDF crystalline lamellae was evaluated using time-resolved WAXD experiments rather than differential scanning calorimetry measurements. Figures $6 \mathrm{~d}-\mathrm{f}$ present examples of WAXD profiles of the neat $i$-PMMA and the blends after both of the crystallization steps were completed. These WAXD profiles were curve separated to crystal peaks and amorphous halos of PVDF and $i$-PMMA. The obtained crystallinities are summarized in Table 1.

Table 1 Crystallinity of the neat polymers and blends

\begin{tabular}{|c|c|c|c|c|}
\hline \multicolumn{2}{|c|}{ Weight fraction } & \multirow[b]{2}{*}{$\mathrm{T}_{C_{-} P V D F} /{ }^{\circ} \mathrm{C}^{\mathrm{a}}$} & \multicolumn{2}{|c|}{$\mathrm{X}_{c}:$ crystallinity/\% } \\
\hline PVDF & i-PMMA & & $P V D F^{\mathrm{b}}$ & $\mathrm{i}-P M M A^{\mathrm{C}}$ \\
\hline 1 & 0 & 148 & 28.9 & - \\
\hline 0.7 & 0.3 & 142 & 27.4 & 11.1 \\
\hline 0.6 & 0.4 & 138 & 25.8 & 23.9 \\
\hline 0.5 & 0.5 & 128 & 25.1 & 31.0 \\
\hline 0.4 & 0.6 & 128 & 21.4 & 47.6 \\
\hline 0 & 1 & - & - & 48.7 \\
\hline
\end{tabular}

Abbreviations: i-PMMA, isotactic-poly(methyl methacrylate); PVDF, poly(vinylidene fluoride). ${ }^{a}$ Crystallization temperatures in the first crystallization step.

${ }^{b}$ Crystallinities normalized to PVDF content in the first crystallization step at indicated $T_{c}$ PVDF ${ }^{\circ}$ Crystallinities normalized to $i$-PMMA content in the second crystallization step at $100^{\circ} \mathrm{C}$.
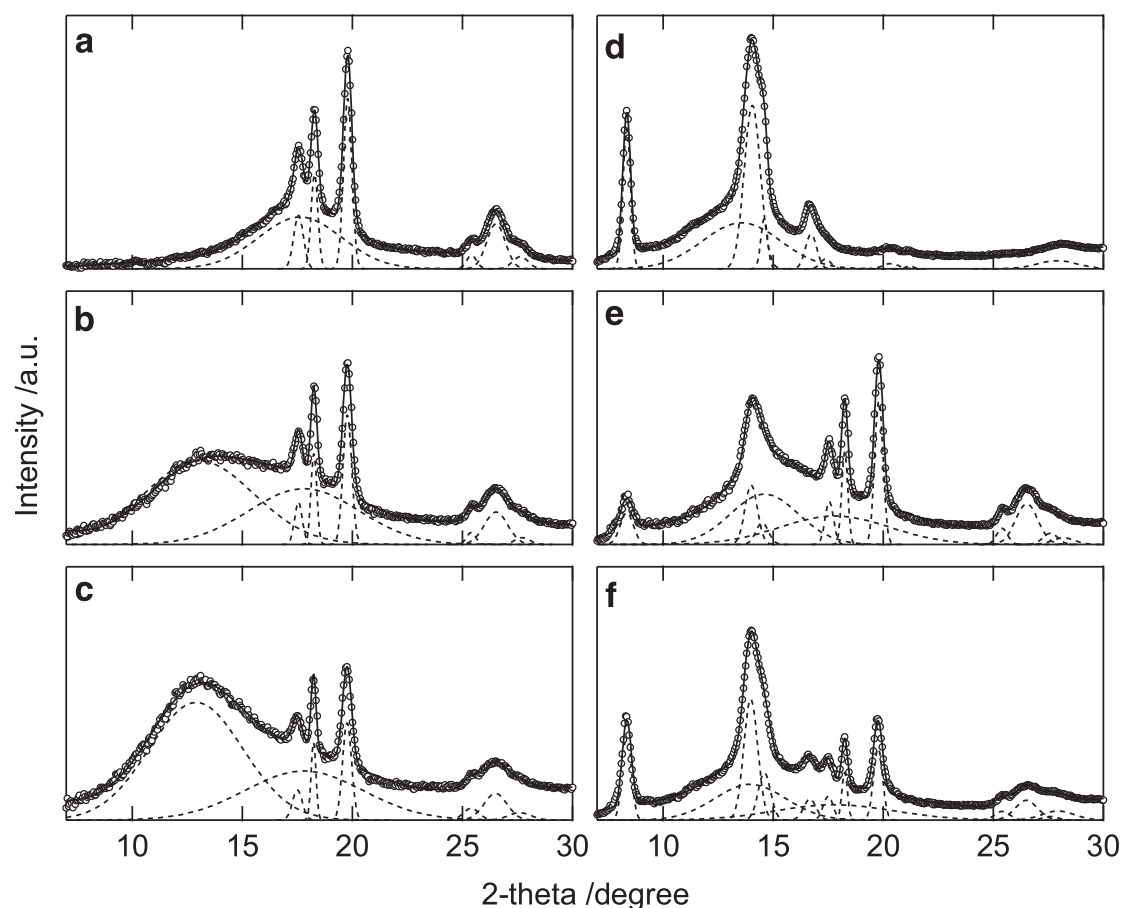

Figure 6 Wide-angle X-ray diffraction (WAXD) patterns for the neat poly(vinylidene fluoride) (PVDF) (a) and the blends that contain 0.6 (b) and 0.4 (c) wt. fr. of PVDF crystallized at $158{ }^{\circ} \mathrm{C}, 138^{\circ} \mathrm{C}$ and $128^{\circ} \mathrm{C}$, respectively, during the first crystallization step and for the neat isotactic-poly(methyl methacrylate) ( $i$-PMMA) (d) and blends that contain 0.6 (e) and 0.4 (f) wt. fr. of PVDF crystallized at $100^{\circ} \mathrm{C}$ during the second crystallization step. 
Figure 7 presents the time evolution of the $i$-PMMA crystallinity during the second crystallization step. It is clearly observed that the time required to reach the maximum crystallinity is considerably shortened in the blends, compared with that in the neat $i$-PMMA, except for the blend that contains 0.7 wt. fr. of PVDF, although the

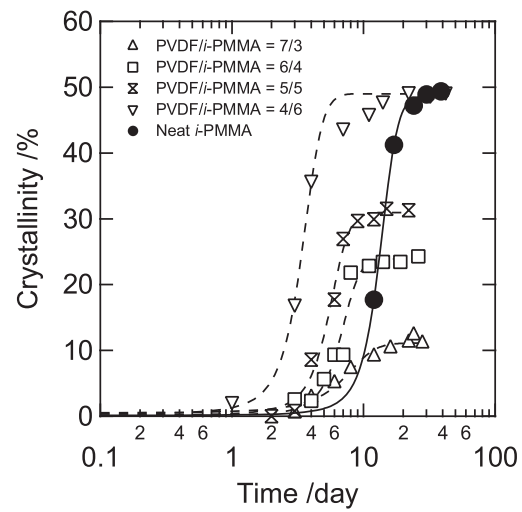

Figure 7 Time evolution of the isotactic-poly(methyl methacrylate) ( $i$-PMMA) crystallinity in the blends during the second crystallization step at $100{ }^{\circ} \mathrm{C}$. crystallization rate usually decreases with increasing counter component content. In this blend system, as shown in Figure 7, the induction period of the crystallization of the blends is shorter than that of the neat $i$-PMMA. Therefore, this unique behavior of the crystallization rate may be attributed to the role of pre-crystallized PVDF as a nucleating agent. In addition, an uncrystallized PVDF part with a lower glass transition temperature may facilitate the flexible movement of the $i$-PMMA chains during their secondary nucleation process.

If the $i$-PMMA component crystallizes between the PVDF crystalline lamellae, the pre-existing PVDF crystal may physically interfere with the subsequent crystal growth of $i$-PMMA. In the crystallization from a restricted space, the Avrami index $n$ has been reported to be considerably reduced ${ }^{33-36}$. The plots of $1-X_{\mathrm{t}}$ vs $\ln t$ based on equation (4) are presented in Figure $3 \mathrm{~b}$. The shape of the plots is almost the same for all the samples including the neat $i$-PMMA, except for the blend that contains 0.7 wt. fr. of PVDF. The slope for this blend is gentler than that for the other samples; namely, $n$ is depressed. Therefore, the crystallization for this blend is confined, which is also reflected in its remarkably low crystallinity (Figure 7 and Table 1). Such confined crystallization of $i$-PMMA in this blend may arise from a small $L 2$, as shown in Figure 5. Although the blend that
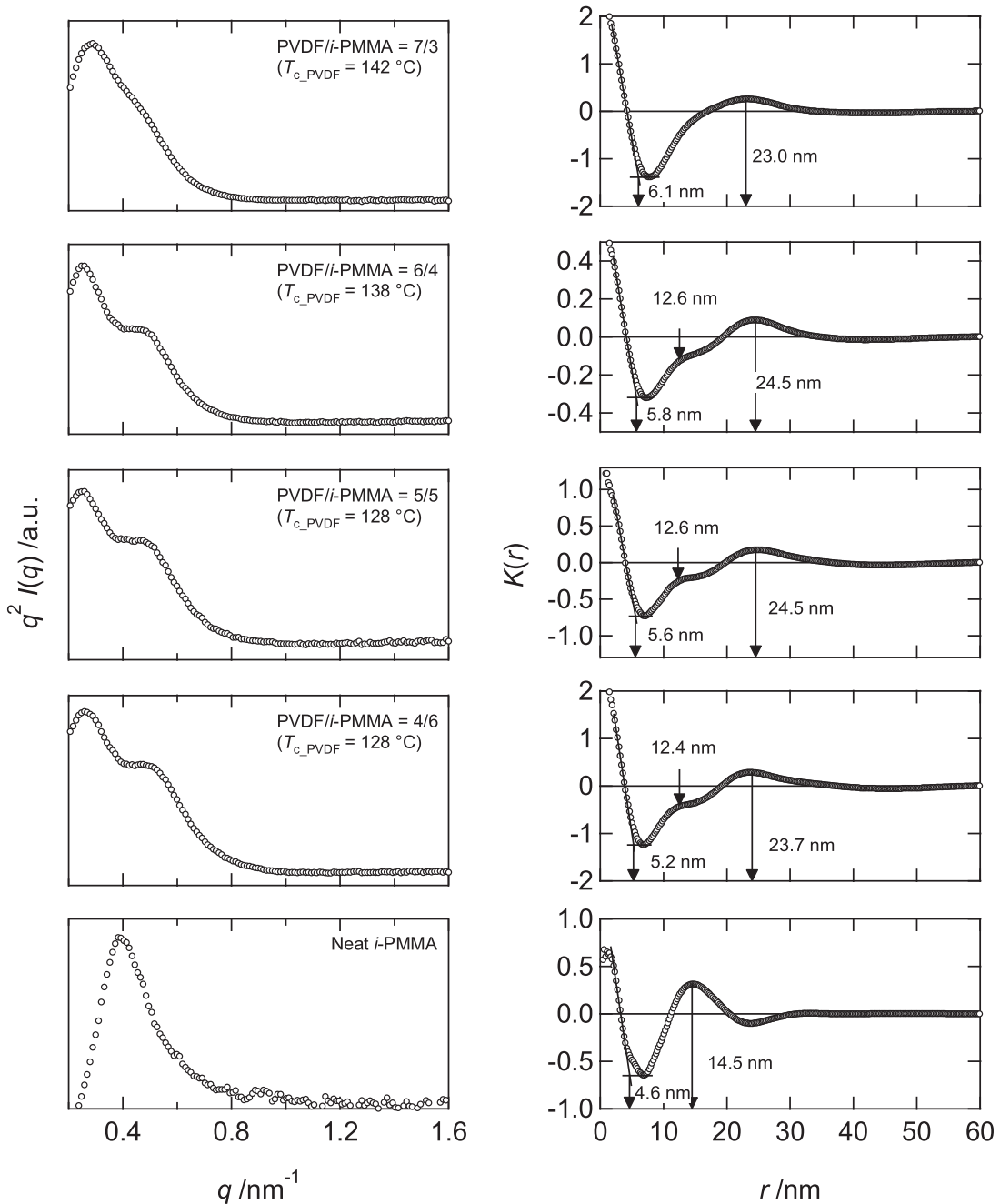

Figure 8 Lorentz-corrected small-angle X-ray scattering (SAXS) profiles (left) and the corresponding one-dimensional correlation functions (right) for the neat isotactic-poly(methyl methacrylate) ( $i$-PMMA) and blends during the second crystallization step. 
contains 0.4 wt. fr. of PVDF exhibits a similar $L 2$, the amount of PVDF in the amorphous layer should be different. The fraction of the PVDF component present in the amorphous layer can be calculated as $50.8 \%$ and $31.4 \%$ for blends with 0.7 and 0.4 wt. fr. of PVDF, respectively, based on the crystallinity in Table 1. Therefore, it is suggested that the crystallization of $i$-PMMA in the blend that contains 0.7 wt. fr. of PVDF is more restricted not only because of space limitations but also by the amorphous PVDF.

Figure 8 presents the Lorentz-corrected SAXS profiles and the onedimensional correlation functions for the neat $i$-PMMA and the blends crystallized during the second crystallization step. Two peaks (one is shoulder-like) can be observed at approximately $q=0.25$ and $0.5 \mathrm{~nm}^{-1}$ in the SAXS profiles of the blends. The time-resolved SAXS profiles during the melting process of the blend that contains $0.6 \mathrm{wt}$. fr. of PVDF are also shown in Figure 9. As shown in Figure 9, the shoulder-like peak at approximately $q=0.5 \mathrm{~nm}^{-1}$ gradually disappears at approximately $140-150{ }^{\circ} \mathrm{C}$; then, the other peak at approximately $q=0.25 \mathrm{~nm}^{-1}$ disappears at approximately $160^{\circ} \mathrm{C}$. As the melting points of the neat $i$-PMMA and PVDF are $144{ }^{\circ} \mathrm{C}$ and $170^{\circ} \mathrm{C}$, respectively, the shoulder-like peak is undoubtedly attributable to the presence of $i$-PMMA crystalline lamellae. The peak position because of the $i$-PMMA crystalline lamellae in the blend (approximately $q=0.5 \mathrm{~nm}^{-1}$ ) is located in the considerably larger $q$ side (which indicates a smaller structure in real space) than that of the neat $i$ PMMA $\left(q=0.4 \mathrm{~nm}^{-1}\right)$ presented in Figure 8. $i$-PMMA should be able to crystallize in two different locations in the blends; these regions include the interlamellar region of the PVDF crystal and other larger regions, such as interfibrillar and interspherulite regions. If $i$ PMMA does not crystallize between the pre-existing PVDF lamellae but instead crystallizes at the other locations, it is difficult to imagine such shrinkage of the structure spacing. This result may strongly support the assumption that $i$-PMMA was crystallized between the PVDF crystalline lamellae.

In the correlation functions of the blends, a crank shape is observed between the first minimum and maximum peaks. Furthermore, the shape becomes clearer with increasing $i$-PMMA content. This type of shape in the correlation function may be explained by assuming a three-phase model, which is schematically illustrated in Figure 10. In this model, the phase (phase A in Figure 10) with the highest density can be undoubtedly assigned to the PVDF crystal because PVDF has the greatest density. A problem arises in determining the phases of $\mathrm{B}$ and $\mathrm{C}$ that can be assigned to the crystalline $i$-PMMA and the amorphous PVDF/i-PMMA mixture, respectively. Normally, phase B, which has the second highest density, is assigned to another crystalline phase (the crystalline $i$-PMMA phase in this case) because the density of the crystalline phase is greater than that of amorphous phase and also because both sides of any crystal phase constitute the amorphous layers. However, the possibility that $i$-PMMA crystallizes at the adjacent phase of the PVDF crystal cannot be completely ruled out, considering that the electron density of PVDF is high even in the amorphous state. If the electron density of the amorphous $\mathrm{PVDF} / i$ PMMA mixture is greater than that of crystalline $i$-PMMA, phase $\mathrm{C}$ would be assigned to the crystalline $i$-PMMA phase. In this case, there should be an electron density step (the same height as phase B) or a relatively gentle gradient between phases A and C. However, no clear evidence supporting that assignment was observed in these SAXS results. Therefore, the clear assignment of phases $\mathrm{B}$ and $\mathrm{C}$ remains ambiguous.

Based on the above model, the total long period $d$ (that is, the spacing between the PVDF crystalline lamellae), the spacing $d^{*}$ between phases $\mathrm{A}$ and $\mathrm{B}$, and the crystalline lamellar thickness of

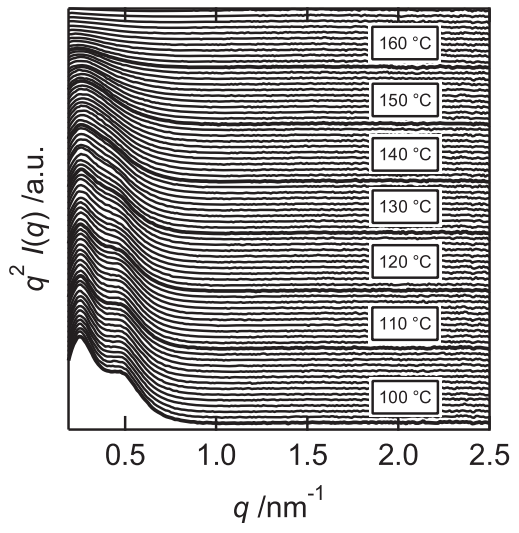

Figure 9 Time-resolved small-angle X-ray scattering (SAXS) profiles in the melting process for the blend that contains $0.6 \mathrm{wt}$. fr. of poly(vinylidene fluoride) (PVDF) first crystallized at $138^{\circ} \mathrm{C}$ during the first crystallization step and then at $100{ }^{\circ} \mathrm{C}$ during the second crystallization step.

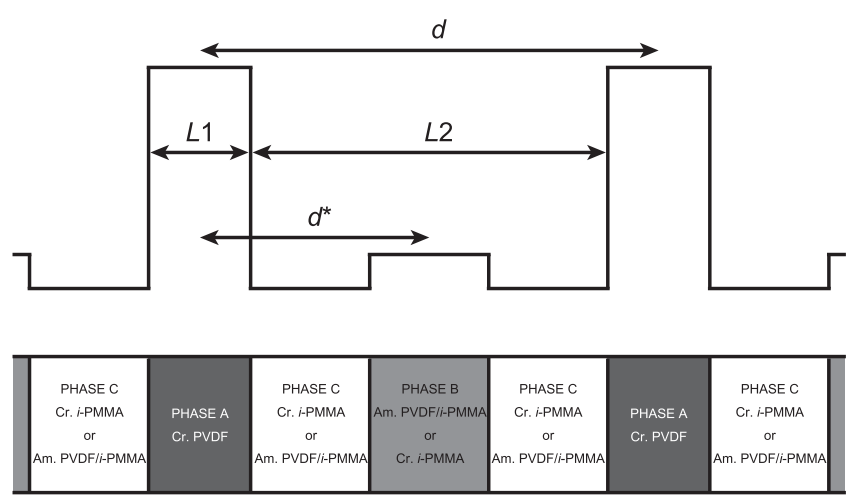

Figure 10 Schematic of the electron density and phase model.

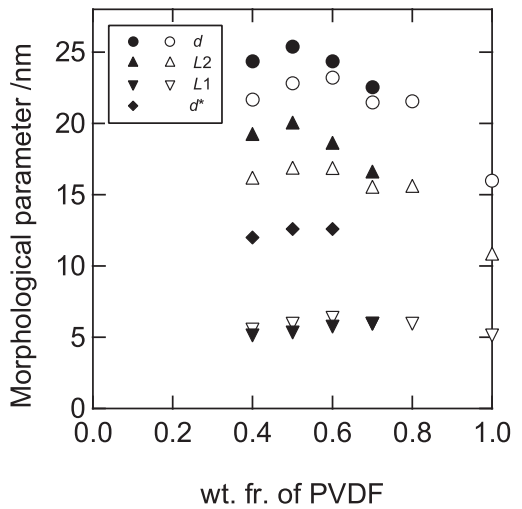

Figure 11 Morphological parameters estimated from the small-angle X-ray scattering (SAXS) peaks and one-dimensional correlation functions during the second crystallization step (filled symbols) and during the first step at

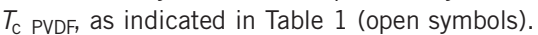

PVDF $L 1$ and $L 2=d-L 1$ were estimated from the one-dimensional correlation functions in Figure 8. Here, the lamellar thickness $L 1$ can be assigned to the crystalline lamellar thickness of PVDF because it exhibits almost the same thickness as that obtained during the first crystallization step. The obtained morphological parameters are plotted in Figure 11, in which the $d$ obtained from $q_{\mathrm{m}}$ was used and the $d^{*}$ of the blend that contained 0.7 wt. fr. of PVDF was not 
estimated, most likely because of the low crystallinity of $i$-PMMA. The morphological parameters obtained during the first crystallization step at $T_{\mathrm{c} \text { PVDF }}$ shown in Table 1 are also replotted in Figure 11. After the second crystallization step, the total long periods $d$ are considerably increased, along with the thickening of $L 2$, especially in the $i$-PMMA-rich blends that contain $0.4,0.5$ and 0.6 wt. fr. of PVDF. As the crystallization of $i$-PMMA should occur between the PVDF crystalline lamellae, this increasing of $L 2$ indicates that $i$-PMMA crystallized at the interlamellar region and expanded the pre-existing PVDF crystalline lamellar structure. The details concerning how the subsequent crystallization alters the pre-existing higher-order structure require further investigation.

\section{CONCLUSIONS}

PVDF in the blends with $i$-PMMA exhibited depressions of both the overall crystallization rate and the melting point depending on the blend composition, as is expected for a typical miscible crystalline/ amorphous blend. This behavior occurs because $i$-PMMA, which has an extremely slow crystallization rate, behaves as an amorphous component during the crystallization of PVDF, even at temperatures less than the $T_{\mathrm{m}}$ of $i$-PMMA. In the PVDF-rich blends, the amorphous $i$-PMMA component included between the PVDF crystalline lamellae increased the spacing. However, the inclusion of $i$-PMMA into the interlamellar region was limited in the PVDF-poor blends, that is, a part of $i$-PMMA was excluded from that space, most likely by decreasing the crystallization rate of PVDF relative to the diffusion rate of $i$-PMMA.

In the subsequent crystallization of $i$-PMMA under the pre-existing PVDF crystal structure, the overall crystallization rate was enhanced, especially in the $i$-PMMA-rich blends. As the induction period for the nucleation of $i$-PMMA in the blends was remarkably decreased compared with that of the neat $i$-PMMA, it was concluded that the pre-existing PVDF crystal acted as a nucleating agent.

The SAXS measurements performed after both steps of crystallization and in the melting process revealed that $i$-PMMA was crystallized between the PVDF crystalline lamellae and that the crystallization of $i$-PMMA expanded the spacing between the PVDF crystalline lamellae. In the blend that contained 0.7 wt. fr. of PVDF, confined crystallization of $i$-PMMA was suggested by the depression of the Avrami index, the lower crystallinity of $i$-PMMA and the narrower space in the crystallizable layer $(L 2)$.

\section{ACKNOWLEDGEMENTS}

The SAXS experiments were performed under the approval of the Photon Factory Program Advisory Committee (proposal no. 2010G528 and 2004G338).

1 Mandelkern, L. Crystallization of Polymers. 2nd edn, Ch. 4 in Vol. 1 (2002) \& Ch. 11 in Vol. 2 (2004) (Cambridge Univ. Press, Cambridge, UK).

2 Russel, T. P., Ito, H. \& Wignall, G. D. Neutron and X-ray-scattering studies on semicrystalline. Polymer blends. Macromolecules 21, 1703-1709 (1988).

3 Russel, T. P. \& Stein, R. S. An investigation of the compatibility and morphology of semicrystalline poly(e-caprolactone)-poly(vinyl chloride) blends. J. Polym. Sci. Part B: Polym. Phys. 21, 999-1010 (1983).

4 Cheung, Y. W., Stein, R. S., Lin, J. S. \& Wignall, G. D. Small-angle scattering investigations of poly( $\varepsilon$-caprolactone)/polycarbonate blends. 2 . Small-angle $\mathrm{x}$-ray and light scattering study of semicrystalline/semicrystalline and semicrystalline/amorphous blend morphologies. Macromolecules 27, 2520-2528 (1994).

5 Chiu, H. J., Chen, H. L. \& Lin, J. S. Crystallization induced microstructure of crystalline/crystalline poly(vinylidenefluoride)/poly(3-hydroxybutyrate) blends probed by small angle X-ray scattering. Polymer (Guildf) 42, 5749-5754 (2001)

6 Liu, L. Z., Chu, B., Penning, J. P. \& John Manley, St. R. Synchrotron SAXS study of miscible blends of semicrystalline poly(vinylidene fluoride) and semicrystalline poly(1,4-butylene adipate). Macromolecules 30, 4398-4404 (1997).
7 Liu, L. Z., Chu, B., Penning, J. P. \& John Manley, St. R. A Synchrotron SAXS study of miscible blends of semicrystalline poly(vinyl-idenefluoride) and semicrystalline poly(1,4-butylene adipate). II. Crystallization, morphology and PBA inclusion in PVF2 spherulites. J. Polym. Sci. Part B: Polym. Phys. 38, 2296-2308 (2000).

8 Keith, H. D. \& Padden, F. J. Spherulitic crystallization from the melt. I. Fractionation and impurity segregation and their influence on crystalline morphology. J. Appl. Phys. 35, 1270-1286 (1964).

9 Saito, H. \& Stuehn, B. Exclusion of noncrystalline polymer from the interlamellar region in poly(vinylidene fluoride)/poly(methyl methacrylate) blends. Macromolecules 27, 216-218 (1994).

10 Okabe, Y., Murakami, H., Osaka, N., Saito, H. \& Inoue, T. Morphology development and exclusion of noncrystalline polymer during crystallization in PVDF/PMMA blends. Polymer (Guildf) 51, 1494-1500 (2010).

11 Martuscelli, E., Pracella, M. \& Yue, W. P. Influence of composition and molecular mass on the morphology, crystallization and melting behaviour of poly(ethylene oxide)/ poly(methyl methacrylate) blends. Polymer (Guildf) 25, 1097-1106 (1984).

12 Zhang, X., Man, S., Han, C. C. \& Yan, D. Nucleation induced by phase separation in the interface of polyolefin blend. Polymer (Guildf) 49, 2368-2372 (2008).

13 Mitra, M. K. \& Muthukumar, M. Theory of spinodal decomposition assisted crystallization in binary mixtures. J. Chem. Phys. 132, 184908 (2010).

14 Arai, F., Takeshita, H., Dobashi, M., Takenaka, K., Miya, M. \& Shiomi, T. Effects of liquid-liquid phase separation on crystallization of poly(ethylene glycol) in blends with isotactic poly(methyl methacrylate). Polymer (Guildf) 53, 851-856 (2012).

15 Ikehara, T. \& Nishi, T. Interpenetrated spherulites of poly(butylene succinate) poly(vinylidene chloride-co-vinyl chloride) blends. An optical microscopic study. Polym. J 32, 683-687 (2000).

16 Hirano, S., Terada, Y., Ikehara, T. \& Nishi, T. Observation of interpenetrated spherulites by confocal laser scanning microscopy. Polym. J. 33, 371-373 (2001).

17 Qiu, Z., Ikehara, T. \& Nishi, T. Unique morphology of poly(ethylene succinate)/ poly(ethylene oxide) blends. Macromolecules 35, 8251-8254 (2002).

18 Hirano, S., Nishikawa, Y., Terada, Y., Ikehara, T. \& Nishi, T. Miscibility and crystallization behavior of crystalline/crystalline polymer blends. poly(ester carbonate)/poly (L-lactic acid). Polym. J. 34, 85-88 (2002).

19 Ikehara, T., Nishikawa, Y. \& Nishi, T. Evidence for the formation of interpenetrated spherulites in poly(butylene succinate-co-butylene carbonate)/poly(I-lactic acid) blends investigated by atomic force microscopy. Polymer (Guildf) 44, 6657-6661 (2003).

20 Ikehara, T., Kimura, H. \& Qiu, Z. Penetrating spherulitic growth in poly(butylene adipate-co-butylene succinate)/poly(ethylene oxide) blends. Macromolecules $\mathbf{3 8}$, 5104-5108 (2005)

21 Ikehara, T., Kurihara, H., Qiu, Z. \& Nishi, T. Study of spherulitic structures by analyzing the spherulitic growth rate of the other component in binary crystalline polymer blends. Macromolecules 40, 8726-8730 (2007).

22 Qiu, Z., Yan, C., Lu, J., Yang, W., Ikehara, T. \& Nishi, T. Various crystalline morphology of poly(butylene succinate-co-butylene adipate) in its miscible blends with poly(vinylidene fluoride). J. Phys. Chem. B 111, 2783-2789 (2007).

23 Ikehara, T., Jinnai, H., Kaneko, T., Nishioka, H. \& Nishi, T. Local lamellar structures in banded spherulites analyzed by three-dimensional electron tomography. J. Polym. Sci. Part B: Polym. Phys. 45, 1122-1125 (2007).

24 Qiu, Z., Yan, C., Lu, J. \& Yang, W. Miscible crystalline/crystalline polymer blends of poly(vinylidene fluoride) and poly(butylene succinate-co-butylene adipate): spherulitic morphologies and crystallization kinetics. Macromolecules 40, 5047-5053 (2007).

$25 \mathrm{Lu}$, J., Qiu, Z. \& Yang, W. Effects of blend composition and crystallization temperature on unique crystalline morphologies of miscible poly(ethylene succinate)/poly(ethylene oxide) blends. Macromolecules 41, 141-148 (2008).

26 Tadokoro, H., Chatani, Y., Kusanagi, H. \& Yokoyama, M. Structure of isotactic poly(methyl methacrylate). Macromolecules 3, $441-447$ (1970).

27 Roerdink, E. \& Challa, G. Influence of tacticity of poly(methyl methacrylate) on the compatibility with poly(vinylidene fluoride). Polymer (Guildf) 19, 173-178 (1978).

28 Sasaki, H., Bala, P. K., Yoshida, H. \& Ito, Eiko. Miscibility of PVDF/PMMA blends examined by crystallization dynamics. Polymer (Guildf) 36, 4805-4810 (1995).

29 High Energy Accelerator Research Organization, Photon Factory. < http://pfwww.kek.jp/>

30 Kobayashi, M., Tashiro, K. \& Tadokoro, H. Molecular vibrations of three crystal forms of poly(vinylidene fluoride). Macromolecules 8, 158-171 (1975).

31 Uemoto, S. \& Okui, N. Master curve of crystal growth rate and its corresponding state in polymeric materials. Polymer (Guildf) 43, 1423-1427 (2002).

32 Strobl, G. R. \& Schneider, M. Direct evaluation of the electron density correlation function of partially crystalline polymers. J. Polym. Sci. Part B: Polym. Phys. 18 1343-1359 (1980).

33 Sakurai, K., MacKnight, W. J., Lohse, D. J., Schulz, D. N. \& Sissano, J. A. Blends of amorphous-crystalline block copolymers with amorphous homopolymers. 2. Synthesis and characterization of poly(ethylene-propylene) diblock copolymer and crystallization kinetics for the blend with atactic polypropylene. Macromolecules 27, 4941-4951 (1994)

34 Shiomi, T., Tsukada, H., Takeshita, K., Takenak, K. \& Tezuka, Y. Crystallization of semicrystalline block copolymers containing a glassy amorphous component. Polymer (Guildf) 42, 4997-5004 (2001).

35 Takeshita, H., Ishii, N., Araki, C., Miya, M., Takenaka, K. \& Shiomi, T. Crystallization and structure formation of block copolymers containing a Glassy amorphous component. J. Polym. Sci. Part B: Polym. Phys. 42, 4199-4206 (2004).

36 Takeshita, H., Gao, Y. J., Natsui, T., Rodriguez, E., Miya, M., Takenaka, K. \& Shiomi, T. Formation of phase structure and crystallization behavior in blends containing polystyrene-polyethylene block copolymers. Polymer (Guildf) 48, 7660-7671 (2007). 